\title{
To Operate or Not to Operate: The Dilemma between Surgery versus Conservative Management of Cervical Disc Herniation
}

\author{
Carlos Perez Gonzalez, MD, Yahira Acevedo-Santiago, MD, Franklin E. Caldera, D0, MBA and *Yejia \\ Zhang, MD, PhD
}

Department of Physical Medicine and Rehabilitation, Perelman School of Medicine, University of Pennsylvania, USA

Received: September 22, 2017; Published: October 04,2017

*Corresponding author: Yejia Zhang, Department of Physical Medicine and Rehabilitation, Perelman School of Medicine, University of Pennsylvania, USA, Email: Yejia.Zhang@uphs.upenn.edu

\section{Case Report}

\section{History and Physical Examination}

A 49 year old male commercial airline pilot presented to the PM\&R spine clinic for an "epidural steroid injection". The patient complained of neck pain radiating to his left posterolateral arm, and to the $4^{\text {th }}$ and $5^{\text {th }}$ digits occasionally. Symptoms began 28 days earlier, and involved waking up in pain, without any history of injury. He described his neck and radiating arm pain as sharp and constant, with an intensity range of 2-8/10. Symptoms were exacerbated by neck movement, while cervical traction and tramadol alleviated the pain temporarily. He also had occasional numbness/tingling of the left $4^{\text {th }}$ and $5^{\text {th }}$ digits. The patient was otherwise healthy. The patient was employed as a commercial airline pilot but was on medical leave due to the severity of his symptoms. Due to the nature of his profession and the concern for the safety of passengers, he was not allowed to go back to work until his condition improved. Physical examination was significant for left deltoid atrophy and subtle shoulder abduction weakness. Spurling's maneuver caused radiating pain to the left posterolateral arm at the elbow level. Neck range of motion was decreased due to pain.

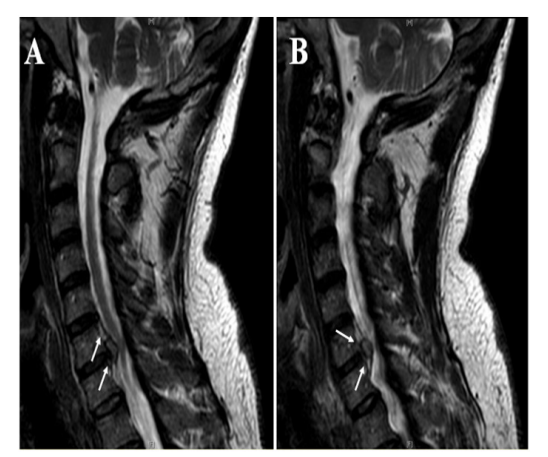

Figure 1 : Sagittal T2-weighted images of the cervical spine. Arrows: herniated/extruded nucleus pulposus. Panels A and B are serial sections.

\section{Imaging Studies}

His primary care physician had requested a cervical spine MRI without intravenous contrast (Figure 1). The most significant finding was a C6-C7 disc bulge (Arrows) with a superimposed moderate-sized left paracentral disc herniation with superior extrusion, which contacted the left anterolateral aspect of the cervical cord and impinged on the exiting left $\mathrm{C} 7$ nerve root.

\section{Clinical Decision Making}

The clinical decision was between continuing conservative care or surgical intervention. Conservative care would have consisted of physical therapy and a cervical epidural steroid injection, using either a transforaminal or an interlaminal approach. In deciding which treatment option to pursue, we considered the following factors: the large size of herniated nucleus pulposus (NP), the severity of the spinal canal stenosis (Figure 1A \& 1B), the risk of further spinal cord compression and neurological consequences, and the patient's occupation. Owing to the nature of his symptoms, including weakness and atrophy of muscles, the patient was referred for surgery. These listed impairments directly affected the patient's work, and we wanted to prevent further deterioration or permanent impairment. A surgical consultation was requested and tramadol was prescribed for pain control.

\section{Surgical Approach}

Four days later, the patient was evaluated by a neurosurgeon and was promptly recommended for C6-C7 anterior cervical discectomy and interbody fusion utilizing a fibular allograft. Surgery was performed 10 days after the patient's initial visit to the PM\&R clinic. The C6-C7 disk space was distracted with a vertebral body spreader. The disk was then removed using a high-speed drill. In dissecting the posterior ligament off the dura, a large free fragment of herniated disk was identified and removed, to decompress the dural sac completely. Foraminotomies were performed bilaterally 
at C6-C7, to provide complete decompression of exiting nerve roots. The C6 and C7 endplates were decorticated and the intervertebral space was filled with allogeneic fibular bone fragments. A metal plate was then contoured between $\mathrm{C} 6$ and $\mathrm{C} 7$ and two screws were inserted into C6 and C7 respectively, to stabilize the two motion segments.

\section{Outcome}

The patient was discharged on the day after the surgery, with home physical therapy and a Philadelphia collar to provide stability of the spine. At 2 weeks after surgery, his neck pain had decreased to $1 / 10$ in intensity, and radiating pain to the left arm had resolved. He returned to work 40 days after the surgery, without any restrictions.

\section{Discussion}

This is a healthy airline pilot, who had an extruded NP, and muscle atrophy. The C5 myotome (Deltoid atrophy) involvement is likely due to extruded NP fragment. The most important clinical decision was whether to inject steroid into the epidural space or to decompress the spinal cord and neuroforamen surgically. Most patients with back and neck pain are treated without surgery, with a combination of physical therapy, medications, and education [1]. Surgical treatments result in superior self-reported disability ratings and less neck pain, but no difference in arm pain between the surgery and conservatively treated groups [2,3]. Our patient depends on his arm and finger dexterity to pilot an airplane. Since the size of the extruded disc was relatively large and the spinal cord was impinged, further neurological damage was likely without surgical decompression. Another major concern is that should we pursue epidural steroid injection, the injected medication may further compress the nerve root and spinal cord. After careful consideration and discussion with the patient, surgery was performed with an excellent outcome.

\section{Author Contribution and Acknowledgements}

Yejia Zhang, MD, PhD has been supported by the Eunice Kennedy Shriver National Institute of Child Health and Human Development (NICHD, 1K08 HD049598). The authors thank Martin F. Heyworth, MD, for critically editing the manuscript and Mr. Robert D. White for valuable insights and discussions. None of the authors have any professional and financial affiliation that could be perceived to be biased in the presentation of the current work.

\section{References}

1. Moss I An HS, Shen F, Li Z, Andersson GBJ, Zhang Y (2014) The non surgical treatment of back pain. In: Irving M. Shapiro and Makarand V. Risbud, ed. The Intervertebral Disc: Molecular and Structural Studies of the Disc in Health and Disease. Springer-Verlag Wien 250-9.

2. Engquist M, Lofgren H, Oberg B, et al. (2017) A 5- to 8-year randomized study on the treatment of cervical radiculopathy: Anterior cervical decompression and fusion plus physiotherapy versus physiotherapy alone. J Neurosurg Spine 26: 19-27.

3. Engquist M, Lofgren H, Oberg B, et al. (2013) Surgery versus nonsurgical treatment of cervical radiculopathy: A prospective, randomized study comparing surgery plus physiotherapy with physiotherapy alone with a 2-year follow-up. Spine (Phila Pa 1976) 38: 1715-1722.

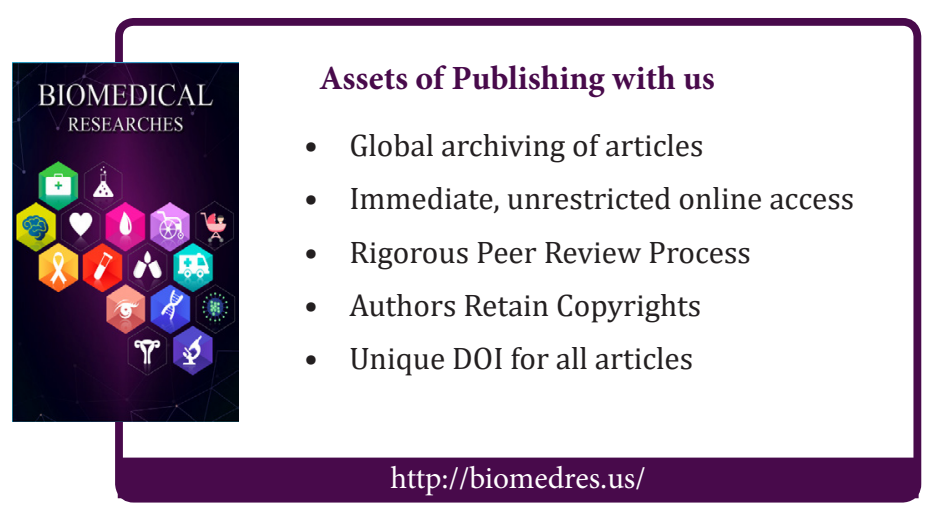

